\title{
Therapeutic management with biological anti-TNF- $\alpha$ agent in severe psoriasis associated with chronic hepatitis B: A case report
}

\author{
ANCA RADUCAN $^{1}$, STEFANA BUCUR ${ }^{2}$, CONSTANTIN CARUNTU ${ }^{3}$, TRAIAN CONSTANTIN ${ }^{4}$, \\ IULIANA ELENA NITA ${ }^{2}$, NICUTA MANOLACHE ${ }^{5}$ and MARIA-MAGDALENA CONSTANTIN ${ }^{2,6}$ \\ ${ }^{1}$ Dr. Anca Răducan Anti-Aging Dermatology Clinic, 900705 Constanta; ${ }^{2}$ The Second Department of \\ Dermatology, Colentina Clinical Hospital, 020125 Bucharest; ${ }^{3}$ Department of Dermatology, \\ Prof. 'N. Paulescu' National Institute of Diabetes, Nutrition and Metabolic Diseases, 021106 Bucharest; \\ ${ }^{4}$ Department of Urology, 'Prof. Dr. Th. Burghele' Hospital, 050659 Bucharest; ${ }^{5}$ Department of Dermatology, \\ Faculty of Pharmacological Sciences, 'Dunarea de Jos' University of Medicine and Pharmacy, 800201 Galati; \\ ${ }^{6}$ The Second Department of Dermatology, 'Carol Davila' University of Medicine and Pharmacy, 050474 Bucharest, Romania
}

Received August 9, 2018; Accepted January 14, 2019

DOI: $10.3892 / \mathrm{etm} .2019 .7567$

\begin{abstract}
Systemic therapy in patients with concurrent psoriasis and chronic hepatitis B is a challenging task for both dermatologists and gastroenterologists since there is a high risk for hepatitis $\mathrm{B}$ virus (HBV) reactivation and hepatic toxicity under biological therapy. The therapeutic management of a patient with psoriasis and infection with the HBV is a challenge as the classical systemic treatment (methotrexate, acitretin, cyclosporine) shows a high risk of immunosuppression and/or hepatic toxicity and the biological therapy is endangered by the possibility of $\mathrm{HBV}$ reactivation. We present the case of a patient with moderate-severe psoriasis and chronic hepatitis B for whom we assessed the risk-benefit relation and considered useful to initiate the anti-TNF therapy concomitantly with the antiviral therapy with entecavir. The therapeutic algorithm included initiation of anti-TNF therapy with etanercept $2 \times 50 \mathrm{mg} /$ week combined with entecavir, an antiviral treatment administered continuously since the diagnosis of the HBV hepatitis, with hepatic function and viral load monitoring. After 3 months of therapy with etanercept the patient was given a dose of etanercept of $50 \mathrm{mg} /$ week combined with entecavir $0.5 \mathrm{mg} /$ day which he continued until week 36 when psoriatic lesions had cleared (PASI=0.6; DLQI=0). No adverse effects were registered and there was no evidence of $\mathrm{HBV}$ viral replication or changes in viral markers. We wish to emphasize that the use of etanercept in a patient with psoriasis and hepatitis B is a successful therapeutic alternative which
\end{abstract}

Correspondence to: Dr Stefana Bucur, The Second Department of Dermatology, Colentina Clinical Hospital, 19-21 Stefan cel Mare Street, 020125 Bucharest, Romania

E-mail: stefanabucur11@gmail.com

Key words: psoriasis, etanercept, chronic hepatitis B, entecavir, anti-TNF- $\alpha$ agent may be safely used concomitantly with entecavir, with regular monitoring of viral load and hepatic function tests.

\section{Introduction}

The therapeutic management of a patient with psoriasis and infection with the hepatitis B virus (HBV) is a challenge as the classical systemic treatment [methotrexate (MTX), acitretin, cyclosporine] shows a high risk of immunosuppression and/ or hepatic toxicity and the biological therapy is endangered by the possibility of $\mathrm{HBV}$ reactivation. We wish to emphasize that the use of etanercept in a patient with psoriasis and hepatitis B is a successful therapeutic alternative which may be safely used concomitantly with entecavir, with regular monitoring of viral load and hepatic function tests.

\section{Case report}

A 38-year old patient diagnosed with psoriasis, presented with moderate-severe psoriasis vulgaris, lesions aggravating in the past few years. The patient followed long-term local treatment with keratolytics, emollients and potent dermocorticoids which led to the occurrence of abdominal stretch marks, but also systemic treatment with MTX and photochemotherapy (PUVA) with unsatisfactory therapeutic effect.

The patient received MTX in a dose of $15 \mathrm{mg} /$ week from March 2004 to June 2004 and 20 mg/week from February 2008 to May 2008 and 20 treatments/month of PUVA therapy between October 1998 and December 1998, both treatments with no therapeutic effect.

The study was approved by the local Ethics Committee of 'Carol Davila' University of Medicine and Pharmacy (Bucharest, Romania), and a signed informed consent was obtained from the patient included in this study.

The dermatological examination upon hospitalization shows the presence of relatively large erythematous-squamous plaques and patches with clearly outlined borders $(5-10 \mathrm{~cm}$ diameter) and tendency to coalescence. The lesions are infil- 


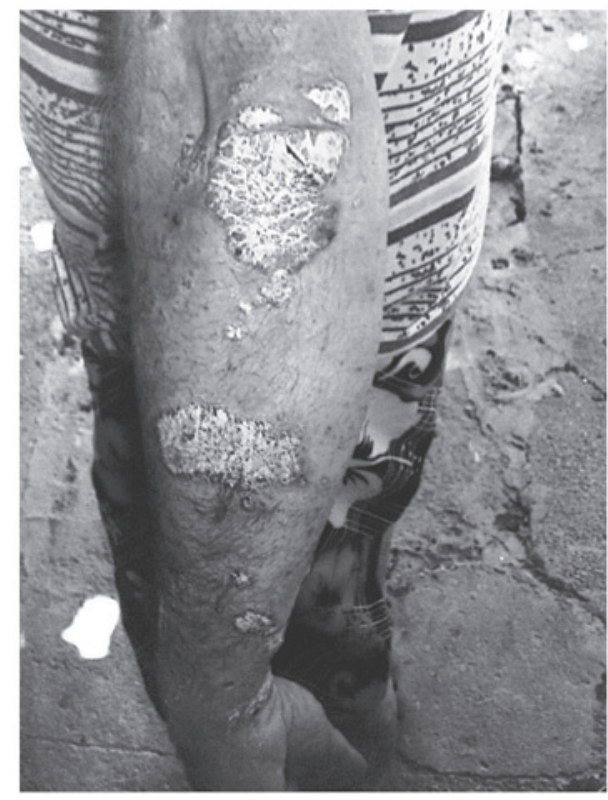

Figure 1. Infiltrated lesions, with thick white scales, easily detachable, distributed over the elbow and forearm.

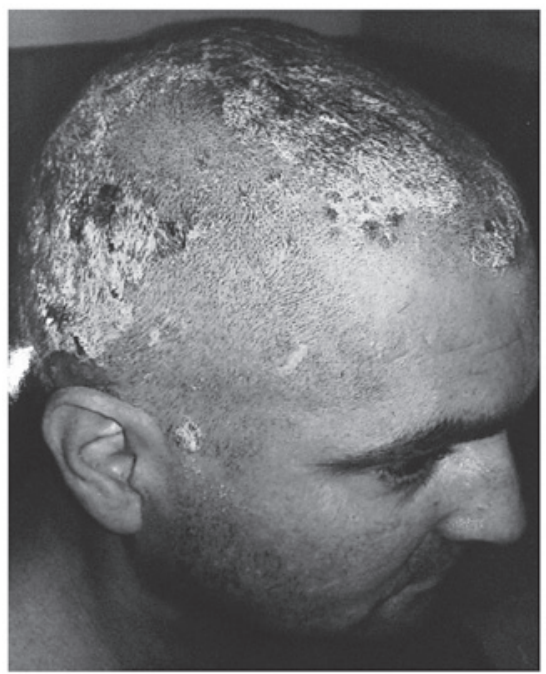

Figure 2. Erythemato-squamous circumscribed plaques, outlined by the hair line.

trated, covered with thick, whitish, easily detachable flakes and are located at the level of the extension areas (elbows, forearms, the back of the hands, knees, before the tibia, lumbosacral) and in the abdominal region (Fig. 1). The scalp is $70 \%$ affected, the circumscribed erythematous-squamous plaques being limited by the hairline and accompanied by moderate itching (Fig. 2). Besides, there are also alterations of the finger and toe nails, with subungual hyperkeratosis, distal onycholysis, pitting, 'oil spot' yellow discoloration and multiple cross lines (Fig. 3). Furthermore, the patient suffers from arthralgia and morning joint stiffness of the hands, elbows and knees.

\section{Results}

Paraclinical investigations included complete blood count, tests to assess hepatic and kidney function, protein electrophoresis,

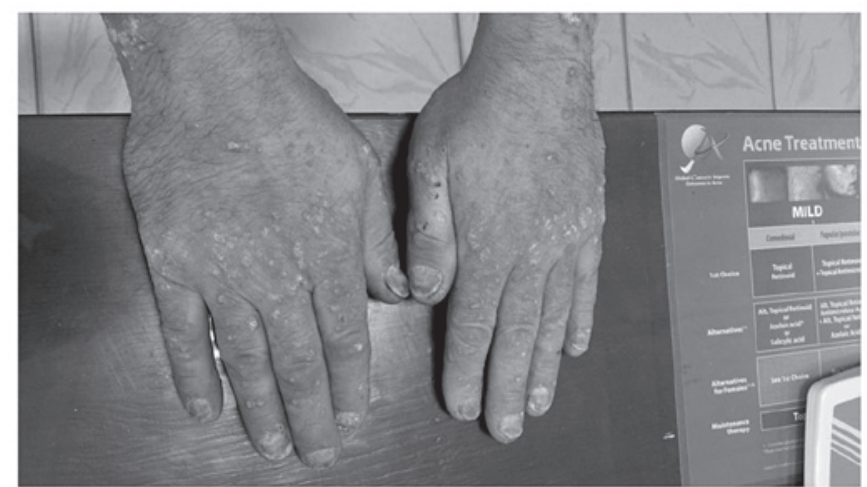

Figure 3. Subungual hyperkeratosis, distal onycholisys, pitting, yellow discoloration and multiple horrizontal lines.

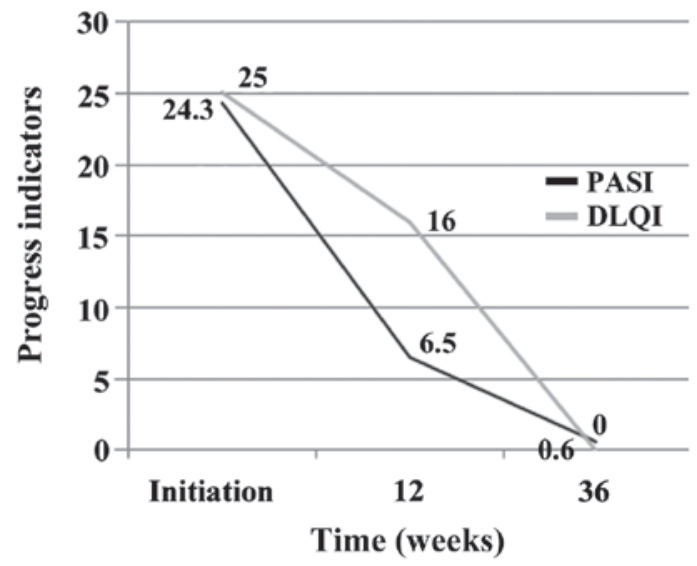

Figure 4. PASI and DLQI in dynamics. PASI, Psoriasis Area and Severity Index; DLQI, Dermatology Life Quality Index.

total cholesterol, urine exam, viral markers for hepatitis B, hepatitis C and HIV, QuantiFERON-TB Gold test (Cellestis Limited, Carnegie, Australia), EKG, BMI, radiography of the hand joint and pulmonary radiography. The results have shown borderline hypercholesterolaemia (210 mg/dl), positive Ag HBs, positive Ac HBs, positive Ag HBe, positive QuantiFERON-TB Gold test, BMI $=29.4 \mathrm{~kg} / \mathrm{m}^{2}$, elevated levels of ESR erythrocyte sedimentation rate (ESR) and C-reactive protein (CRP) and normal transaminases.

The radiographies showed marginal bone erosions in both hands and knees, bone proliferation and right wrist fusion. After pneumology examination the chemical prophylaxis was initiated for tuberculosis with isoniazid $300 \mathrm{mg} /$ day for 9 months, but after 1 month of chemotherapy, the therapy for psoriasis was started. Additionally, the patient was diagnosed with chronic HBV hepatitis with positive $\mathrm{Ag} \mathrm{HBe}$ (positive Ag HBs, positive Ag HBe, negative IgG anti HVD, PCR ADN-VHB=2341650). Fibromax assessment of fibrosis and inflammatory activity revealed minimum fibrosis at FibroTest (score 0.31) and minimum steatosis at SteatoTest (score 0.30) (both from BioPredictive, Paris, France). Subsequently, we initiated treatment with entecavir $0.5 \mathrm{mg} / \mathrm{day}$.

In this context, the patient was diagnosed with moderately-severe psoriasis vulgaris [Psoriasis Area and Severity Index $(\mathrm{PASI})=24.3$; Dermatology Life Quality 


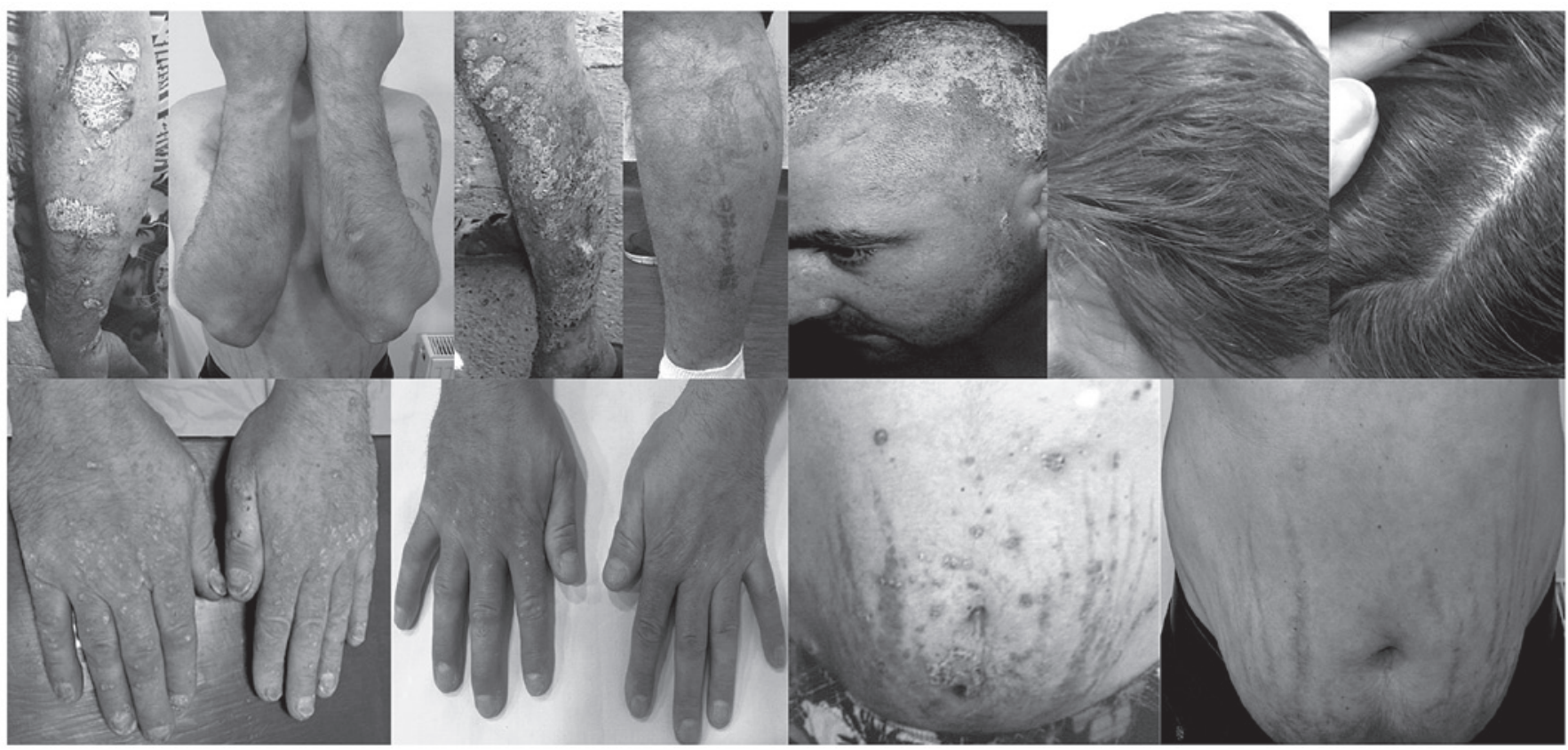

Figure 5. The evolution of psoriatic lesions.

Index (DLQI)=25], chronic HBV hepatitis with positive $\mathrm{Ag} \mathrm{HBe}$ and overweight (BMI=29.4). The therapeutic algorithm included initiation of anti-TNF therapy with etanercept (2x50 mg/week) combined with entecavir, an antiviral treatment administered continuously since the diagnosis of the HBV hepatitis, with hepatic function and viral load monitoring. Patient reached PASI 75 at week 12 and registered significant improvement of DLQI (Fig. 4) and decrease of viral load (below the detection limit of 20 copies/ml), however, without changes in viral markers. After 3 months of therapy with etanercept the patient was given a dose of etanercept of $50 \mathrm{mg} /$ week combined with entecavir $0.5 \mathrm{mg} /$ day which he continued until week 36 when psoriatic lesions had cleared (PASI=0.6; DLQI=0). The patient had normal levels of ESR, CRP, including transaminases after the cure with MTX.

A previous interesting study indicated that an increase in malondialdehyde (MDA) level and a decrease in antioxidants levels are features of psoriasis and that MTX seems to have protective antioxidative properties during the treatment of psoriasis (1).

No adverse effects were registered and there was no evidence of $\mathrm{HBV}$ viral replication or changes in viral markers. The patient is continuing the combined therapy at the present time with very good results and the evolution can be observed in Fig. 5. All tests above are performed regularly. They are in normal ranges and the HBV DNA is undetectable.

\section{Discussion}

Our patient was diagnosed clinically in 1998 and histopathologically in 2014. A cutaneous punch-biopsy was performed and the histopathology results revealed hyperkeratosis and parakeratosis with neutrophils inclusions between keratin blades, wide areas of agranulocytosis, moderate acanthosis with relatively uniformly elongated and thickened epidermal cristae, increased mitotic keratinocyte activity and moderate perivascular inflammatory infiltrate around dilated capillaries. The histopathological aspect confirmed the clinical diagnosis of psoriasis vulgaris.

There are specific dermoscopic features suggestive for psoriasis and reflectance confocal microscopy (RCM) enables identification of most of the histological features of psoriatic lesions and both dermoscopy and RCM allow a real-time non-invasive examination of skin lesions offering the possibility to perform serial determinations (2).

RCM can also successfully evaluate dilated and tortuous dermal capillaries, which represent a histological feature of psoriasis vulgaris. Compared with the skin of healthy subjects, capillaries examined in lesions of psoriasis have higher mean values of micromorphological parameters (area, perimeter, Feret's diameter) and that for each papilla, the number of capillary sections is higher (3).

As for the treatment, recent studies argue that in moderately-severe unstable psoriasis, with low DLQI or therapeutic failure of other systemic treatments, the use of anti-TNF- $\alpha$ agents in patients with an HBV infection could be an option, but it requires a multidisciplinary approach and especially a good collaboration with the enterologist (4-7).

At present, antiviral treatment of adult patients with chronic viral hepatitis B in Romania includes entecavir, adefovir, lamivudine or pegylated interferon $\alpha-2 \mathrm{a}$ (8). Entecavir is a guanosine nucleoside analogue active against HBV polymerase, from the class of nucleoside and nucleotide reverse transcriptase inhibitors, and through its antiviral action, it inhibits the synthesis of AND HBV. Entecavir, the first line of monotherapy treatment and long-term use in chronic HBV hepatitis, may be administered in doses of 0.5 or $1 \mathrm{mg} /$ day with regular 6 months assessments; the treatment may be continued depending on the biochemical and virologic response until the occurrence of Ac HBs (8).

Recent studies underline the association of anti-TNF agents with increased risk of viral reactivation in patients with 
inactive chronical HBV infection $(9,10)$. Data from the literature indicate a higher incidence of infections and lymphoma in patients treated with infliximab and adalimumab as compared to etanercept. Also, the general safety profile of etanercept when it comes to the risk of infection and hepatotoxicity in patients with psoriasis, psoriatic arthritis and rheumatoid arthritis is higher than that of the other anti-TNF- $\alpha$ agents (11).

Biosimilars could be a treatment option, but unfortunately, at present there is rather limited evidence provided by the clinical trials, which would help a dermatologist feel more comfortable about prescribing biosimilars. After the studies ended, we can conclude that there is no specific safety concern today that was raised and no specific lack of efficacy either. There are also ongoing centered postmarketing studies regarding biosimilar immunogenicity which is the main concern for biosimilars (12).

The maximum estimated risk of reactivation of HBV in patients with history of hepatitis B who did not receive any biological therapy for psoriasis for an average follow-up period of 30 months was of $2.7 \%$ according to a 2015 study (13). In a different study carried out on a group of 468 patients with negative $\mathrm{Ag} \mathrm{HBs}$ and positive Ac HBc treated with anti-TNF agents for rheumatoid conditions, HBV reactivation was reported for $1.7 \%$ of patients and viral markers were monitored during anti-TNF treatment (14). In the case of our patient, no HBV reactivation was recorded at 9 months after initiation of treatment with etanercept and entecavir.

Treatment of patients with psoriasis and chronic HBV hepatitis with etanercept administered concomitantly with lamivudine or entecavir has proven to be safe and it reduces the risk of reactivation of HBV hepatitis (15).

Studies having shown reactivation of HBV after anti-TNF- $\alpha$ therapy in patients with inactive hepatitis B (negative Ag HBs) support the prophylactic use of antiviral agents for HBV carriers even if hepatic function is within normal limits or viral load is undetectable (16).

During the first 12 weeks after the initiation of therapy, etanercept may be administered in different doses, either $50 \mathrm{mg}$ twice a week or $50 \mathrm{mg}$ once a week - both therapeutic schemes being efficient. Nevertheless, the rate of therapeutic response at week 24 is higher if etanercept is initially administered with $50 \mathrm{mg}$ doses twice a week; after week 24 the treatment may be administered either continuously or intermittently, both cases showing long and mid-term efficiency $(17,18)$. In our case, we followed the treatment protocol approved for Romania with an initial dose of $2 \times 50 \mathrm{mg} /$ week followed by a usual dose of $50 \mathrm{mg} /$ week combined with entecavir $0.5 \mathrm{mg} /$ day with good results, as shown by the dynamics of PASI and DLQI and hepatic function.

Recent studies show good adherence to etanercept treatment in patients with psoriasis, higher in the case of intermittent treatment than in continuous treatment, which suggests that the intermittent therapy with etanercept could be the best therapeutic option both clinically and in terms of adherence $(19,20)$.

The Medical Council of the National Psoriasis Foundation of America recommends triple serology (Ag HBs, Ac HBs, $\mathrm{Ac} \mathrm{HBc}$ ) for hepatitis B screening before starting therapy with anti-TNF agents, but also with other immunosuppressive drugs such as ustekinumab, cyclosporine or MTX (21).
Ustekinumab can be an effective alternative therapy for moderate to severe psoriasis because the benefit-risk profile in the studied group was favorable, consistent with the global studies of ustekinumab, but further additional studies are needed to evaluate long-term administration and the safety profile (22).

Psoriasis has mast cell activation as a key mechanism. The important role of mast cells in stress-induced activation of skin inflammation is suggested by their strategic location near cutaneous nerve structures and blood vessels and their complex interconnections with nerve fibers, immune cells and keratinocytes. An interesting study shows that in the hairy skin acute stress induces an increased mast cell degranulation that persists even after prolonged exposure to stress, while in glabrous skin a short-term stress exposure has a strong stimulating effect of mast cell degranulation that subsides in intensity as exposure to stress persists (23).

For treatment, we can use acitretin in combination with biologic therapies because it is not immunosuppressive. A large number of observations indicate that psoriasis is linked to plasma membrane alterations in different types of cells. Some studies showed that acitretin, even at low concentrations, is capable of inducing the reduction of red cell deformability (24).

For the patients with psoriatic arthritis, the heterogeneity and complexity of psoriatic arthritis pathogenesis requests complex methodological approaches. Going deeper in the pathogenesis of this disease, the associated genetic predisposition can lead to new immune biomarkers whether from the proteomic or genomic area and identify new therapeutic approaches (25).

Many systemic therapies available for the management of psoriasis patients who cannot be treated with more conservative options, such as topical agents and/or phototherapy, can worsen or reactivate a chronic infection. Therefore, before administering immunosuppressive therapies it is mandatory to screen patients for some infections, including hepatitis B virus.

For our patient, etanercept was chosen due to its high efficiency and a very good safety profile with low immunogenicity and intermittent administration in selected cases.

Biologic therapy did not cause HBV reactivation. However, there are potentially serious complications associated with HBV reactivation and it is important to measure viral load in patients with a history of HBV infection prior to initiation of biologic therapy to rule out occult carriage. These patients should also be monitored regularly together with a hepatologist (26).

In conclusion, by presenting this case we wish to emphasize that the use of etanercept in a patient with psoriasis and hepatitis B is a successful therapeutic alternative which may be safely used concomitantly with entecavir, with regular monitoring of viral load and hepatic function tests.

\section{Acknowledgements}

Not applicable.

\section{Funding}

No funding was received. 


\section{Availability of data and materials}

The datasets used and/or analyzed during the current study are available from the corresponding author on reasonable request.

\section{Authors' contributions}

MMC contributed to the design and conception of the study, and revised it carefully for important intellectual content. $\mathrm{SB}, \mathrm{CC}, \mathrm{TC}, \mathrm{NM}$ were responsible for acquiring the data by screening the papers identified on Pubmed. SB revised the study critically for important intellectual content. CC, TC, $\mathrm{NM}$ were involved in drafting the study. IEN analyzed and interpreted the data. AR contributed to the conception and design of the study, she revised the language and contributed to drafting the study. All authors agreed to be accountable for all aspects of the work in ensuring that questions related to the accuracy or integrity of any part of the work are appropriately investigated and resolved. All authors read and approved the final manuscript.

\section{Ethics approval and consent to participate}

This study was approved by the local Ethics Committee of 'Carol Davila' University of Medicine and Pharmacy (Bucharest, Romania), and a signed informed consent was obtained from the patient included in this study.

\section{Patient consent for publication}

The patient provided written informed consent for the publication of any associated data and accompanying images.

\section{Competing interests}

The authors declare that they have no competing interests.

\section{References}

1. Boda D, Negrei C, Nicolescu F and Balalau C: Assessment of some oxidative stress parameters in methotrexate treated psoriasis patients. Farmacia 62: 704-710, 2014.

2. Căruntu C, Boda D, Căruntu A, Rotaru M, Baderca F and Zurac S: In vivo imaging techniques for psoriatic lesions. Rom J Morphol Embryol 55 (Suppl): 1191-1196, 2014.

3. Batani A, Brănișteanu DE, Ilie MA, Boda D, Ianosi S, Ianosi G and Caruntu C:' Assessment of dermal papillary and microvascular parameters in psoriasis vulgaris using in vivo reflectance confocal microscopy. Exp Ther Med 15: 1241-1246, 2018.

4. Nosotti L, Francesconi F, Izzi S, Berardesca E, Morrone A and Bonifati C: Safety of antitumour necrosis factor- $\alpha$ therapy in psoriatic patients with hepatitis $\mathrm{B}$ virus infection. Br J Dermatol 162: 1408-1410, 2010.

5. Fotiadou C, Lazaridou E and Ioannides D: Safety of anti-tumour necrosis factor- $\alpha$ agents in psoriasis patients who were chronic hepatitis B carriers: A retrospective report of seven patients and brief review of the literature. J Eur Acad Dermatol Venereol 25 471-474, 2011

6. Cassano N, Mastrandrea V, Principi M, Loconsole F, De Tullio N, Di Leo A and Vena GA: Anti-tumor necrosis factor treatment in occult hepatitis B virus infection: A retrospective analysis of 62 patients with psoriatic disease. J Biol Regul Homeost Agents 25: 285-289, 2011

7. Prignano F, Ricceri F, Pescitelli L, Zanieri F and Lotti T: Tumour necrosis factor- $\alpha$ antagonists in patients with concurrent psoriasis and hepatitis B or hepatitis C: A retrospective analysis of 17 patients. Br J Dermatol 164: 645-647, 2011.
8. Romanian Therapeutic Guidelines: Chronic Hepatitis. http://www. ms.ro/wp-content/uploads/2018/08/Anexa_Ordin_protocoale_29_ august_2018.pdf.

9. Zoulim F and Durantel D: Antiviral therapies and prospects for a cure of chronic hepatitis B. Cold Spring Harb Perspect Med 5: a021501, 2015. doi: 10.1101/cshperspect.a021501.

10. Seto WK: Hepatitis B virus reactivation during immunosuppressive therapy: Appropriate risk stratification. World J Hepatol 7: 825-830, 2015.

11. Domm S, Cinatl J and Mrowietz U: The impact of treatment with tumour necrosis factor-alpha antagonists on the course of chronic viral infections: A review of the literature. Br J Dermatol 159: 1217-1228, 2008

12. Girolomoni G, Altomare G, Ayala F, Berardesca E, Calzavara-Pinton P, Chimenti S, Peserico A, Puglisi Guerra A and Vena GA: Safety of anti-TNFa agents in the treatment of psoriasis and psoriatic arthritis. Immunopharmacol Immunotoxicol 34: 548-560, 2012.

13. Olteanu R, Zota A and Constantin MM: Biosimilars: An update on clinical trials (review of published and ongoing studies). Acta Dermatovenerol Croat 25: 57-66, 2017.

14. Sanz-Bueno J, Vanaclocha F, García-Doval I, Torrado R, Carretero G, Daudén E, Patricia Ruiz-Genao D, AlsinaGibert MM, Pérez-Zafrilla B, Pérez-Rial G, et al; members of the BIOBADADERM group: Risk of reactivation of hepatitis $B$ virus infection in psoriasis patients treated with biologics: A retrospective analysis of 20 cases from the BIOBADADERM Database. Actas Dermosifiliogr 106: 477-482, 2015.

15. Lee YH, Bae SC and Song GG: Hepatitis B virus (HBV) reactivation in rheumatic patients with hepatitis core antigen (HBV occult carriers) undergoing anti-tumor necrosis factor therapy. Clin Exp Rheumatol 31: 118-121, 2013.

16. Kuroda T, Wada Y, Kobayashi D, Sato H,Murakami S, Nakano M and Narita I: Effect of etanercept and entecavil in a patient with rheumatoid arthritis who is a hepatitis B carrier: A review of the literature. Rheumatol Int 32: 1059-1063, 2012.

17. Chung SJ, Kim JK, Park MC, Park YB and Lee SK: Reactivation of hepatitis B viral infection in inactive HBsAg carriers following anti-tumor necrosis factor-alpha therapy. J Rheumatol 36: 2416-2420, 2009.

18. Strohal R, Chimenti S, Vena GA and Girolomoni G: Etanercept provides an effective, safe and flexible short- and long-term treatment regimen for moderate-to-severe psoriasis: A systematic review of current evidence. J Dermatolog Treat 24: 199-208, 2013.

19. Strohal R, Puig L, Chouela E, Tsai TF, Melin J, Freundlich B, Molta CT, Fuiman J, Pedersen R and Robertson D: The efficacy and safety of etanercept when used with as-needed adjunctive topical therapy in a randomised, double-blind study in subjects with moderate-to-severe psoriasis (the PRISTINE trial). J Dermatolog Treat 24: 169-178, 2013.

20. Esposito M, Gisondi P, Cassano N, Babino G, Cannizzaro MV, Ferrucci G, Chimenti S and Giunta A: Treatment adherence to different etanercept regimens, continuous vs. intermittent, in patients affected by plaque-type psoriasis. Drug Dev Res 75 (Suppl 1): S31-S34, 2014.

21. Babino G, Esposito M, Mazzotta A, Chimenti S and Giunta A: Entecavir and intermittent etanercept therapy in a patient with concurrent hepatitis B virus infection and psoriasis. Acta Derm Venereol 93: 373-374, 2013

22. Olteanu R, Constantin MM,Zota A, Dorobantu DM, Constantin T, Șerban ED, Bălănescu P, Mihele D and Gheucă Solovăstru L: Original clinical experience and approach to treatment study with interleukin 12/23 inhibitor in moderate-to-severe psoriasis patients. Farmacia 64: 918-921, 2016.

23. Căruntu C, Boda D, Musat S, Căruntu A and Mandache E: Stress-induced mast cell activation in glabrous and hairy skin. Mediators Inflamm 2014: 105950, 2014.

24. Negrei C, Arsene AL, Toderescu CD, Boda D and Ilie M: Acitretin treatment in psoriasis may influence the cell membrane fluidity. Farmacia 60: 767-771, 2012.

25. Caruntu C, Boda D, Dumitrascu G, Constantin C and Neagu M: Proteomics focusing on immune markers in psoriatic arthritis. Biomarkers Med 9: 513-528, 2015.

26. Motaparthi K, Stanisic V, Van Voorhees AS, Lebwohl MG and Hsu S; Medical Board of the National Psoriasis Foundation: From the Medical Board of the National Psoriasis Foundation: Recommendations for screening for hepatitis B infection prior to initiating anti-tumor necrosis factor-alfa inhibitors or other immunosuppressive agents in patients with psoriasis. J Am Acad Dermatol 70: 178-186, 2014. 